\title{
Numerical Analysis of Passively Mode-Locked Quantum-Dot Lasers With Absorber Section at the Low-Reflectivity Output Facet
}

\author{
Hercules Simos, Mattia Rossetti, Christos Simos, Charis Mesaritakis, Tianhong Xu, \\ Paolo Bardella, Ivo Montrosset, Member, IEEE, and Dimitris Syvridis
}

\begin{abstract}
In this paper, we present a theoretical study on the optimization of passively mode-locked quantum dot lasers based on an alternative cavity design. In particular, we investigate a geometry in which the saturable absorber is located near the low reflection facet of the chip (output facet). The investigation is carried out by means of a time-domain traveling wave numerical model for quantum-dot active medium for both the gain and absorbing sections. The analysis shows superior performance in terms of pulsewidth and peak power of devices based on the new geometry compared to devices based on the conventional geometry, where the saturable absorber is placed near the high reflectivity facet. The optimization relies on the enhanced bleaching of the saturable absorber when the latter is located near the output facet, which prevents the generation of colliding or self-colliding pulse effects.
\end{abstract}

Index Terms-Mode locking, quantum dots, semiconductor lasers.

\section{INTRODUCTION}

$\mathbf{T}$ WO-SECTION monolithic mode-locked (ML) lasers based on the Quantum-Dot (QD) material system are well-established devices as pulse sources for telecommunications, imaging applications, combining the small footprint and high peak power at multi-Gigabit rates [1], [2]. In the last years, various QD ML configurations have been extensively

Manuscript received June 15, 2012; revised August 31, 2012; accepted September 26, 2012. Date of publication October 3, 2012; date of current version November 28, 2012. This work was supported by the Project FASTDOT, an Integrated Program funded by the European Commission through the 7th ICT-Framework Program, under Grant 224338.

H. Simos is with the Department of Informatics and Telecommunications, Optical Communications Laboratory, National and Kapodistrian University of Athens, Athens GR-15784, Greece, and also with the Department of Electronics, Technological Educational Institute of Piraeus, Athens 12244, Greece (e-mail: simos@di.uoa.gr).

M. Rossetti, T. Xu, P. Bardella, and I. Montrosset are with the Dipartimento di Elettronica e Telecomunicazioni, Politecnico di Torino, Torino 10129, Italy (e-mail: mattia.rossetti@polito.it; tianhong.xu@polito.it; paolo.bardella@polito.it; ivo.montrosset@polito.it).

C. Simos is with the Department of Electronics, Technological Educational Institute of Lamia, Lamia GR-35100, Greece, and also with the Department of Informatics and Telecommunications, Optical Communications Laboratory, National and Kapodistrian University of Athens, Athens GR-15784, Greece (e-mail: simos@teilam.gr).

C. Mesaritakis and D. Syvridis are with the Department of Informatics and Telecommunications, Optical Communications Laboratory, National and Kapodistrian University of Athens, Athens GR-15784, Greece (e-mail: cmesar@di.uoa.gr; dsyvridi@di.uoa.gr).

Color versions of one or more of the figures in this paper are available online at http://ieeexplore.ieee.org.

Digital Object Identifier 10.1109/JQE.2012.2222352 investigated and optimized in terms of the generated pulse characteristics. Compared to active or hybrid mode locking, passive mode-locking, employing a saturable absorber incorporated into the laser, is a simpler and cost effective technique to generate ultra-short pulses, without the need for a radiofrequency $(\mathrm{RF})$ source [2]. Usually, the optimization of such devices is achieved exploiting the coherent interaction of counter-propagating optical pulses in the saturable absorber (colliding-pulse effects) [3]. In the standard configuration, the absorber section is placed near the high reflectivity (HR) cavity mirror in order to enhance the absorption saturation on the basis of a self-colliding pulse effect (self-colliding pulse modelocking, SCPM) [3]. Recently, further optimization on the ML characteristics was theoretically investigated for a QW-based device, exploiting an anti-colliding geometry (anti-colliding pulse mode locking, ACPM), e.g. the absorber section is assumed near the low-reflection coated facet [4]. It was shown that the proposed design leads to substantial increase in output power and a reduction in amplitude and timing jitter. Although in principle this design could also be applied in the case of a QD passively ML laser, its effectiveness, the degree of optimization and the corresponding operating conditions are under question, due to the inherent fundamental differences of the QD lasers compared to QW lasers (the energy bandstructure and the absorption and gain saturation energies).

In this paper we investigate theoretically the optimization of two-section passively ML QD lasers exploiting two alternative designs. In particular, we compare the operation of devices based on the conventional high reflection located absorber geometry, with the alternative geometry in which the absorber is located near the low reflection facet (output) of the laser in terms of average and peak power, pulse width and amplitude noise. We show that this configuration exhibits shorter pulses with higher peak power than the conventional case, but the overall performance is limited in some cases by strong amplitude fluctuations. Furthermore, drawbacks of this configuration and a comparison with the results obtained for QW ML lasers reported in [4] are discussed. The investigation is carried out by means of a finite-difference traveling-wave (FDTW) numerical model. First, a brief description of this numerical model and the simulated devices is given in section II. Detailed results for the comparison of the two considered geometries are presented, analyzed and discussed in section III. Section IV comprises the conclusion of our work. 


\section{Device Modeling}

The numerical model used in the simulations is a finitedifference traveling-wave (FDTW) model for two-section QD ML lasers similar to the one used in [5]. This model is based on the solution of travelling wave equations for the forward and backward propagating fields in the saturable gain/absorbing media and thus it properly accounts for the colliding effects in the absorber. The QD material system is described with a four-energy level system: the separateconfinement hetero-structure layer $(\mathrm{SCH})$, the wetting layer (WL), the first excited state (ES) and the ground state (GS). Compared to [5], the second excited state $\left(\mathrm{ES}_{2}\right)$ and the corresponding transitions are omitted as a means to reduce the simulation time. The inhomogeneous energy distribution fabrication-induced gain broadening is described with a set of multi-population rate equations, accounting only for the electron dynamics. The carrier rate equations for the gain sections read:

$$
\begin{aligned}
\frac{d N_{S C H}}{d t}= & \eta \frac{J}{q} w d z-\frac{N_{S C H}}{\tau_{r, S C H}}-\frac{N_{S C H}}{\tau_{S C H-W L}}+\frac{N_{W L}}{\tau_{W L-S C H}} \\
\frac{d N_{W L}}{d t}= & \frac{N_{S C H}}{\tau_{S C H-W L}}-\frac{N_{W L}}{\tau_{W L-S C H}}-\frac{N_{W L}}{\tau_{r, W L}} \\
& -\frac{N_{W L}}{\tau_{W L-E S}} \sum_{j=1}^{N} G_{E S}^{j}\left(1-\rho_{E S}^{j}\right)+\sum_{j=1}^{N} \frac{N_{E S}^{j}}{\tau_{E S-W L}^{j}} \\
\frac{d N_{E S}^{j}}{d t}= & \frac{G_{E S}^{j} N_{W L}}{\tau_{W L-E S}}\left(1-\rho_{E S}^{j}\right)-\frac{N_{E S}^{j}}{\tau_{E S-W L}^{j}}-\frac{N_{E S}^{j}}{\tau_{E S-G S}} \\
& \left(1-\rho_{G S}^{j}\right)+\frac{N_{G S}^{j}}{\tau_{G S-E S}^{j}}\left(1-\rho_{E S}^{j}\right)-\frac{N_{E S}^{j}}{\tau_{S p o n, E S}} \\
& -\frac{N_{E S}^{j} \rho_{E S}^{j}}{\tau_{A u g, E S}}+\frac{2 d z}{\hbar \omega_{E S}^{j}} \Im\left(E_{E S}^{+}\left(P_{E S}^{+, j}\right)^{*}+E_{E S}^{-}\left(P_{E S}^{-, j}\right)^{*}\right)
\end{aligned}
$$

$$
\begin{aligned}
\frac{d N_{G S}^{j}}{d t}= & \frac{N_{E S}^{j}}{\tau_{E S-G S}}\left(1-\rho_{G S}^{j}\right)-\frac{N_{G S}^{j}}{\tau_{G S-E S}^{j}}\left(1-\rho_{E S}^{j}\right) \\
& -\frac{N_{G S}^{j}}{\tau_{\text {spon }, G S}}-\frac{N_{G S}^{j} \rho_{G S}^{j}}{\tau_{A u g, G S}} \\
& +\frac{2 d z}{\hbar \omega_{G S}^{j}} \Im\left(E_{G S}^{+}\left(P_{G S}^{+, j}\right)^{*}+E_{G S}^{-}\left(P_{G S}^{-, j}\right)^{*}\right) .
\end{aligned}
$$

$N_{S C H}, N_{W L}$ are the total carrier number in the $\mathrm{SCH}$ and the 2-D quantum well (QW) states (wetting layer, WL); $N_{E S}^{j}, N_{G S}^{j}$ are the carrier number of each QD confined state (excited, ground state respectively). All carrier populations are considered for a given longitudinal section of gain section. $E_{E S}^{ \pm}$and $E_{G S}^{ \pm}$corresponds to the forward and backward propagating field for the ES and GS respectively. $\rho_{E S}^{j}, \rho_{G S}^{j}$ are the carrier occupation probability for the ES and GS of the j-th QD group, rescpectively [5]. $G_{n}^{j}$ is the inhomogeneous broadening factor for state $\mathrm{n}(\mathrm{n}=\mathrm{ES}, \mathrm{GS})$ and for the $\mathrm{j}$-th QD group, assuming a Gaussian distribution [5]. The symbol $\Im()$ represents the imaginary part of a complex number. The summations run over the considered number of QD groups $(\mathrm{N}=13)$.

The time constants $\tau_{S C H-W L}$ and $\tau_{W L-E S}$ are the characteristic carrier relaxation times between from $\mathrm{SCH}$ to the QW states (WL) and from the WL to the QD excited states, respectively; $\tau_{E S-G S}^{j}=\tau_{E S-G S}$ is the relaxation time from the ES to GS in the j-th QD group, respectively. The escape times $\tau_{W L-S C H}, \tau_{E S-W L}^{j}$ and $\tau_{G S-E S}^{j}$ are calculated from the capture/relaxation times as:

$$
\begin{aligned}
\tau_{W L-S C H} & =\tau_{S C H-W L} \frac{D O S_{W L} N_{l}}{D O S_{S C H} h_{S C H}} e^{\frac{\Delta E_{S C H-W L}}{k T}} \\
\tau_{E S-W L}^{j} & =\tau_{W L-E S} \frac{D_{E S} N_{d}}{D O S_{W L}} e^{\frac{\Delta E_{W L-E S}^{j}}{k T}} \\
\tau_{G S-E S}^{j} & =\tau_{E S-G S} \frac{D_{G S}}{D_{E S}} e^{\frac{\Delta E_{E S-G S}^{j}}{k T}}
\end{aligned}
$$

where $\triangle E_{S C H-W L}$ is the energy difference between $\mathrm{SCH}$ and $\mathrm{QW}$ band edges and $\Delta E_{W L-E S}^{j}$ is the energy difference between the QW band edge and the excited confined state of the j-th QD group. $D_{E S}, D_{G S}$ are the degeneracy of ES and GS, and $D O S_{S C H}, D O S_{W L}$ are the effective density of states in the $\mathrm{SCH}$ and QW, respectively [5].

The rate equations for the absorber section are enhanced with terms which account for the electron escape processes due to the application of an electric field (thermionic escape and tunneling processes). The corresponding expressions can be found in [5].

The solution of the optical field propagation equation is given by a finite difference scheme for the forward and backward field as in [5]. In comparison to [5], the optical field equation has been split in two uncoupled equations, one for the field at the GS wavelength $\left(E_{G S}\right)$ and one for the field resonant with the ES $\left(E_{E S}\right)$ :

$$
\begin{aligned}
E_{n}^{ \pm}(z+d z, t+d t)= & E_{n}^{ \pm}(z, t)-\frac{1}{2} a_{\text {loss }} E_{n}^{ \pm}(z, t) d z \\
& -i P_{n}^{ \pm}(z, t) d z+S_{\text {spon }, n}^{ \pm}(z, t) d z
\end{aligned}
$$

where $\mathrm{n}=\mathrm{GS}$, ES. $P_{n}^{ \pm}(z, t)$ is the effective polarization for each state and direction of propagation, calculated as:

$$
\begin{aligned}
P_{n}^{ \pm}(z, t) & =\sum_{j=1}^{N} P_{n}^{ \pm, j}(z, t) \\
& =\sum_{j=1}^{N} i \frac{\Gamma \omega_{n, 0}}{\hbar 2 \pi c n_{g} h_{W L}} D_{n} N_{d} G_{n}^{j} A_{n}\left(2 \rho_{n}^{j}-1\right) I_{n}^{ \pm, j}(z, t)
\end{aligned}
$$

$I_{n}^{ \pm, j}(z, t)$ is the convolution integral of the polarization with the field and it is calculated numerically using equations (17)-(18) in [5]. $S_{\text {spon, },}^{ \pm}(z, t)$ represents the contribution of the spontaneous emission noise and its implementation is given by equations (19), (20) and (22) in [5], neglecting the summation for GS/ES. Equation (3) is solved separately for the two states ( $\mathrm{n}=\mathrm{GS}$, ES), taking small enough time sample $(\mathrm{dt}=50 \mathrm{fs})$ to ensure enough gain bandwidth for each state. This approach relaxes the requirements for ultrashort time step for the simulation of the QD gain spectrum, since the entire wide spectrum is considered as two distinct bands [6], [7]. 


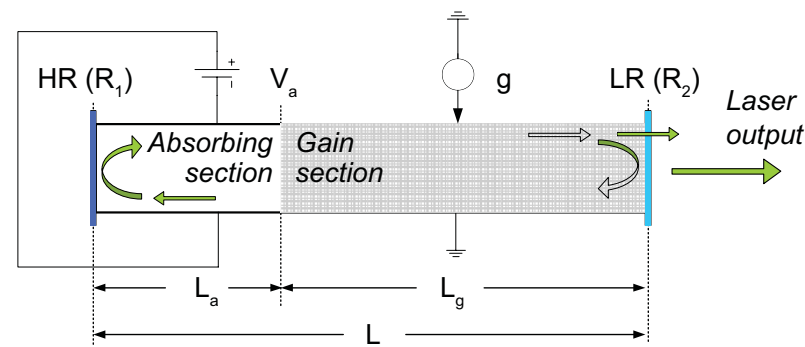

(a)

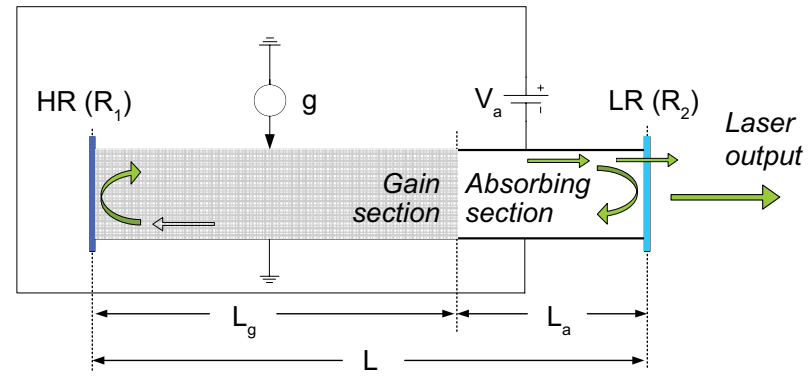

(b)

Fig. 1. Schematic of the monolithic passive mode-locked quantum-dot laser geometries, with the absorber near the (a) HR-facet and (b) LR-facet.

In the numerical model there is no additional non-linear gain saturation coefficient since the spectral hole burning (SHB) non-linear gain compression which dominates in QD system (fast non-linearity) is inherently included through the multiple population rate-equation system used (one rate equation for each QD energy/spectral group and confined state).

The considered device is a two section Fabry-Perot laser with 5 InAs quantum dot layers embedded in InGaAs/GaAs QWs with the peak gain for the GS at $1.3 \mu \mathrm{m}$. The total length of the laser cavity (L) is $2 \mathrm{~mm}$ which results to a repetition frequency of $22.4 \mathrm{GHz}$, and the length of the absorbing section $\left(\mathrm{L}_{a}\right)$ is $0.3 \mathrm{~mm}$. The high reflection $(\mathrm{HR})$ facet reflectivity is $R_{1}=0.99$ and the low reflection (LR) facet reflectivity is $R_{2}=0.1$. The physical and structural model parameters are given in table I and they have been previously used for the modeling of real devices with very good agreement between simulation and experiments [5]. Some parameters such inhomogeneous gain broadening and gain coefficients have been extracted by fitting experimentally obtained gain spectra. Capture and relaxation time have been extracted in order to reproduce pump and probe experiments [5]. In order to be aligned with usual experimental practice, pulse duration is evaluated from the full width at half maximum (FWHM) of the mathematically calculated autocorrelation trace (AC). We followed this approach because the autocorrelation is always symmetric and insensitive to strong pulse shape irregularities which can lead to errors in the calculation of real pulse width [8]. All the calculations have been performed over a long pulse series and after the complete development of ML, rejecting the initial transients.

Fig. 1 illustrates the ML configurations considered in this study. In the typical configuration (top), the absorber is located near the high-reflection facet, enabling the selfcolliding effects for an enhanced saturation of the absorber
TABLE I

PhysicAl AND STRUCTURAL PARAMETERS USED IN CALCULATIONS

\begin{tabular}{|c|c|c|}
\hline Parameter & Symbol & Value \\
\hline Cavity length & $L$ & $2 \mathrm{~mm}$ \\
\hline Absorber length & $L_{a}$ & $0.3 \mathrm{~mm}$ \\
\hline Waveguide width & $w$ & $6 \mu \mathrm{m}$ \\
\hline $\begin{array}{l}\text { Thickness of the active } \\
\text { region }(\mathrm{SCH})\end{array}$ & $h_{S C H}$ & $435 \mathrm{~nm}$ \\
\hline $\begin{array}{l}\text { Thickness of the quantum } \\
\text { well (WL) }\end{array}$ & $h_{W L}$ & $5 \mathrm{~nm}$ \\
\hline $\begin{array}{l}\text { Quantum dot surface } \\
\text { density }\end{array}$ & $N_{d}$ & $2.8 \times 10^{14} \mathrm{~m}^{-2}$ \\
\hline Confinement factor & $\Gamma$ & 0.067 \\
\hline Quantum dot layers & $N_{l}$ & 5 \\
\hline Refractive index & $n_{g}$ & 3.35 \\
\hline Waveguide loss coefficient & $a_{\text {loss }}$ & $150 \mathrm{~m}^{-1}$ \\
\hline $\begin{array}{l}\text { Facet power reflectivities } \\
(\mathrm{HR}, \mathrm{LR})\end{array}$ & $R_{1}, R_{2}$ & {$[0.99,0.1]$} \\
\hline Degeneracy of GS, ES & $D_{G S}, D_{E S}$ & {$[2,4]$} \\
\hline $\begin{array}{l}\text { Interband transition matrix } \\
\text { element for GS, ES }\end{array}$ & $A_{G S}, A_{E S}$ & {$[5.8,6] \times 10^{-27} \mathrm{~m}^{3} \mathrm{eV}$} \\
\hline $\begin{array}{l}\text { Relaxation time from WL } \\
\text { to ES }\end{array}$ & $\tau_{W L-E S}$ & $0.4 \mathrm{ps}$ \\
\hline $\begin{array}{l}\text { Relaxation time from ES } \\
\text { to GS }\end{array}$ & $\tau_{E S-G S}$ & $0.4 \mathrm{ps}$ \\
\hline $\begin{array}{l}\text { Spontaneous recombination } \\
\text { time for GS, ES }\end{array}$ & $\begin{array}{c}\tau_{\text {spon }, G S} \\
\tau_{\text {spon }, E S}\end{array}$ & {$[2,2] \mathrm{ns}$} \\
\hline $\begin{array}{l}\text { Non radiative } \\
\text { recombination time for } \\
\text { SCH, WL }\end{array}$ & $\begin{array}{c}\tau_{r, S C H} \\
\tau_{r, W L}\end{array}$ & {$[0.2,0.2] \mathrm{ns}$} \\
\hline $\begin{array}{l}\text { Auger recombination time } \\
\text { constant for GS, ES }\end{array}$ & $\begin{array}{c}\tau_{\text {Aug, GS }} \\
\tau_{\text {Aug, ES }}\end{array}$ & {$[0.275,0.660] \mathrm{ps}$} \\
\hline $\begin{array}{l}\text { Energy difference between } \\
\text { WL and ES }\end{array}$ & $\Delta E_{W L-E S}^{j}$ & $82 \mathrm{meV}$ \\
\hline $\begin{array}{l}\text { Energy difference between } \\
\text { continuum and WL }\end{array}$ & $\Delta E_{S C H-W L}$ & $111.8 \mathrm{meV}$ \\
\hline $\begin{array}{l}\text { Full width at half } \\
\text { maximum of } \\
\text { inhomogeneous broadening }\end{array}$ & $\Gamma_{i n h}$ & $34 \mathrm{meV}$ \\
\hline $\begin{array}{l}\text { Full width at half } \\
\text { maximum of homogeneous } \\
\text { broadening }\end{array}$ & $2 \hbar \Gamma_{\text {hom }}$ & $15 \mathrm{meV}$ \\
\hline $\begin{array}{l}\text { Spontaneous emission } \\
\text { coupling factor }\end{array}$ & $\beta$ & $10^{-4}$ \\
\hline $\begin{array}{l}\text { Interband transition energy } \\
\text { for GS, ES }\end{array}$ & $E_{0, n}$ & {$[0.975,1.038] \mathrm{eV}$} \\
\hline
\end{tabular}

(HR configuration). In the alternative geometry (bottom) the absorber is located near the low reflection facet (output) of the laser thus preventing the generation of colliding effects (LR configuration).

\section{RESULTS AND DISCUSSION}

\section{A. Comparison of the HR and LR Configurations: FOM}

In order to compare the efficiency of the HR- and LRabsorber configurations, we performed a complete mapping of the ML operation in terms of average power, peak power 

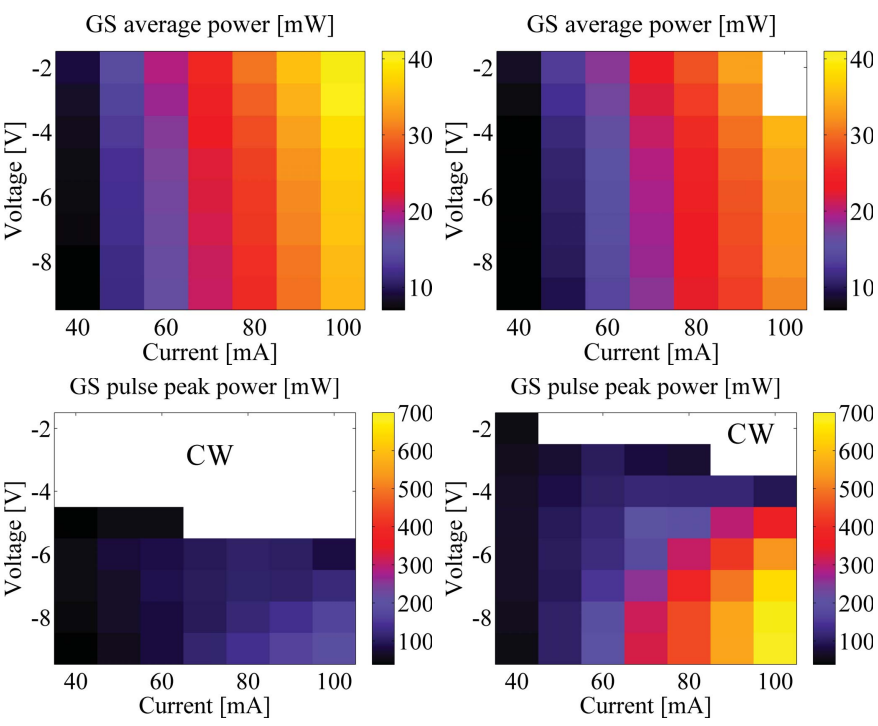

GS pulse peak power $[\mathrm{mW}]$

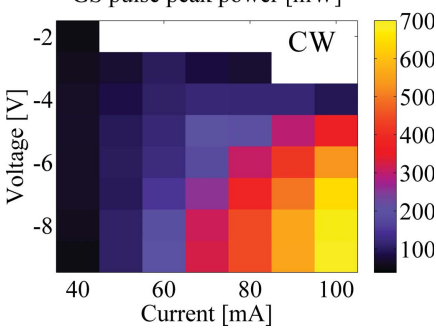

Pulse AC width [ps]

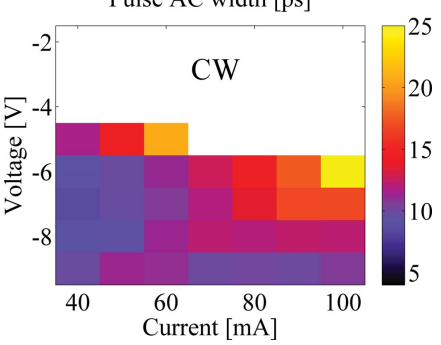

(a)

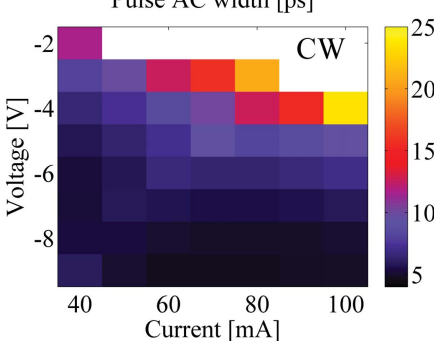

(b)
Fig. 2. Maps of the average power, peak power, and autocorrelation pulsewidth for the considered configurations. (a) HR. (b) LR.

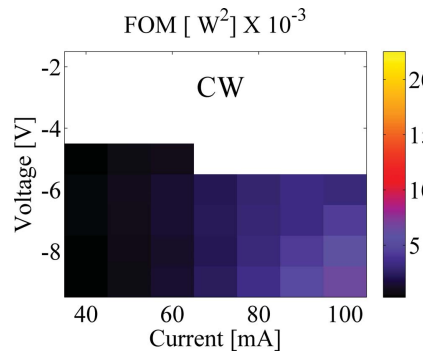

(a)

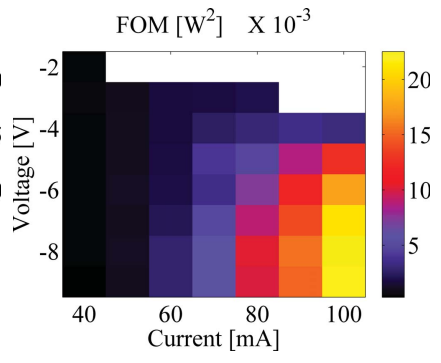

(b)
Fig. 3. Maps of the figure of merit parameter (FOM) for the considered configurations. (a) HR. (b) LR.

and pulsewidth. The maps are shown in Fig. 2. As means to reduce the plots and give the results in a more presentable way, we have calculated the figure of merit (FOM) parameter which is given by the product of average power and peak power (in $\mathrm{W}^{2}$ ). Fig. 3 illustrates FOM as a function of the gain current and the absorber voltage for both the HR and LR geometries. It is easily observed that the LR geometry has superior performance with respect to the HR geometry. First, the LR device exhibits a lower voltage threshold of the ML operation for all currents. Second, the LR device gives significantly higher FOM especially well above the ML current threshold. This is the result of the significantly shorter (and thus stronger) pulses generated by the LR device in this regime, as observed in Fig. 2. In order to clarify the dynamics which lead to the above results, we have plotted

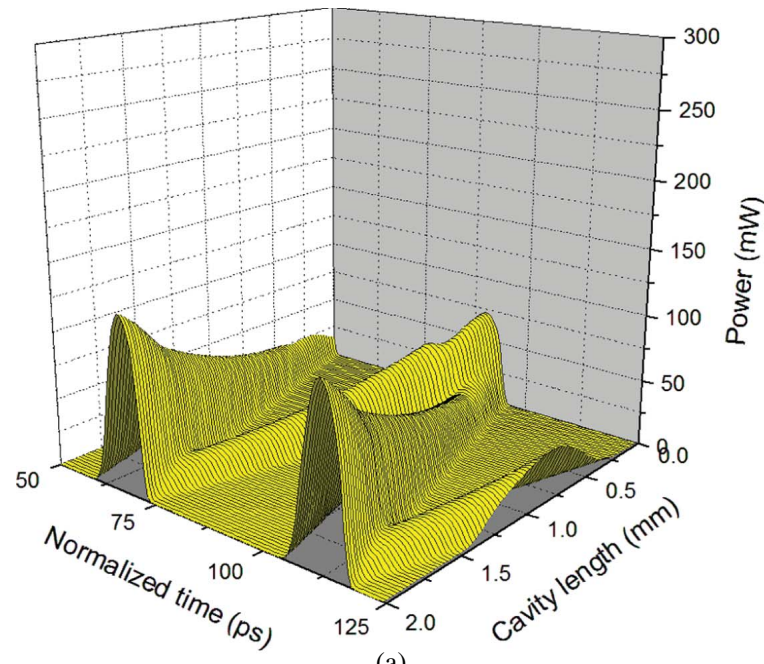

(a)

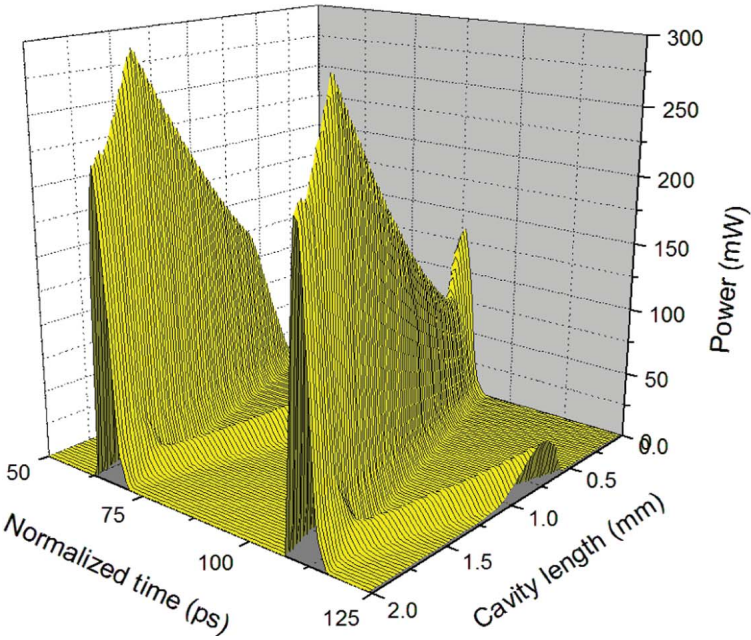

(b)

Fig. 4. Intra-cavity pulse time-space evolution for the two considered configurations. (a) HR. (b) LR.

an instance of the pulse formation inside the laser cavity for both geometries (Fig. 4) for the same operating conditions $\left(\mathrm{I}_{g}=70 \mathrm{~mA}, \mathrm{~V}_{a}=-6 \mathrm{~V}\right)$. It is evident that in the case of the LR geometry, the power impinging at the input of the absorber section (Fig. 4 bottom, at $\mathrm{z}=1.7 \mathrm{~mm}$ ) is much higher than the corresponding power in the HR case (Fig. 4 top, at $\mathrm{z}=0.3 \mathrm{~mm}$ ). This can be explained as follows. In the LR case the field propagates twice along the length of the gain section with a negligible mirror loss (at $\mathrm{z}=0$ ) before it reaches the absorber at the LR facet $(\mathrm{z}=1.7 \mathrm{~mm})$; in the HR case on the contrary, the pulse amplification in the gain section is interrupted by the absorber near the HR facet from one side $(\mathrm{z}=0.3 \mathrm{~mm})$ and by the LR facet from the other side of the cavity $(\mathrm{z}=2 \mathrm{~mm})$. Consequently, in the LR configuration the absorber exhibits enhanced saturation (due to higher power) which enables the generation of shorter pulses with higher peak power than in the HR case. The large difference in FOM shown in Fig. 3 is due to the shorter pulses generated in the LR case. Furthermore, the extended ML operation range in the LR case is due to the earlier saturation of the absorber (e.g. at lower voltage). 
Concluding, although the original idea of utilizing HR design is to enhance the absorption saturation in the SA through a self-colliding effect (the pulse propagates twice in the SA without experiencing significant loss at the HR facet), the power impinging the absorber from the gain section is not that high. The simulation results indicate that in the LR configuration, the coupling of the two strong forward and backward propagating pulses is avoided, but the power impinging the absorber from the gain section is now much larger thus enhancing the absorption saturation and pulse shaping compared to the HR design.

\section{B. Noise and ML Stability}

Besides pulsewidth and peak power, the amplitude noise and the stability of ML operation are very critical for many applications. Fig. 5 shows the maps of the pulse peak power fluctuation for the considered geometries, defined as the ratio of the standard deviation of peak power to the average peak power. Contrary to the FOM performance, at moderate and high gain current values the HR configuration exhibits less noise than the LR one. A combination of the FOM maps with the pulse peak power fluctuation maps of the previous paragraph, leads to the following conclusion: in the regime where the LR configuration exhibits the maximum advantage in terms of FOM compared to the HR configuration, the ML operation suffers from stronger peak power fluctuation than the HR configuration. This gets clearer observing the FOM maps on the bottom of Fig. 5 which include only the conditions with fluctuation lower than 5\%. In order to explain this behavior we focused on the gain and absorption dynamics in different regimes. As described in detail in [5], considering a reference framework moving at the group velocity of the ML pulse, the round trip gain $R(\tau, f)$ experienced by the pulse in the laser cavity is calculated as a function of the optical frequency, $f$, and the relative pulse time, $\tau$ (equation (31) in [5]). $\tau$ is defined as $\tau=t \mp z / v_{g}$ where $v_{g}$ is the group velocity, $\mathrm{z}$ is the spatial coordinate, $\mathrm{t}$ is the absolute time and the sign depends on whether the pulse is propagating in the forward or backward direction. From this coordinate transformation, the round trip gain, $R(\tau, f)$, seen by the pulse can be calculated by integrating over the spatial coordinate $\mathrm{z}$, the time-dependent net gain/absorption spectra experienced by the pulse during both the forward and backward propagation in the cavity (QD gain/absorption spectra can be directly obtained from the imaginary part of the QD polarization (4)).

As explained in detail in [5], the time-frequency representation of the QD gain in the cavity and consequently of $R(\tau, f)$ is justified since an adiabatic approximation has been introduced to describe the dependence of the QD polarization on the time-dependent occupation probabilities of the QD states reported in (4).

Maps of the normalized round trip gain, $\ln (R(\tau, f)) / \mathrm{L}$, for two different regimes in the HR cavity design, are shown in Fig. 6, where the ML pulse envelope and the corresponding optical spectrum (in linear scale) are also shown. The area enclosed by the black line in the center of the map corresponds
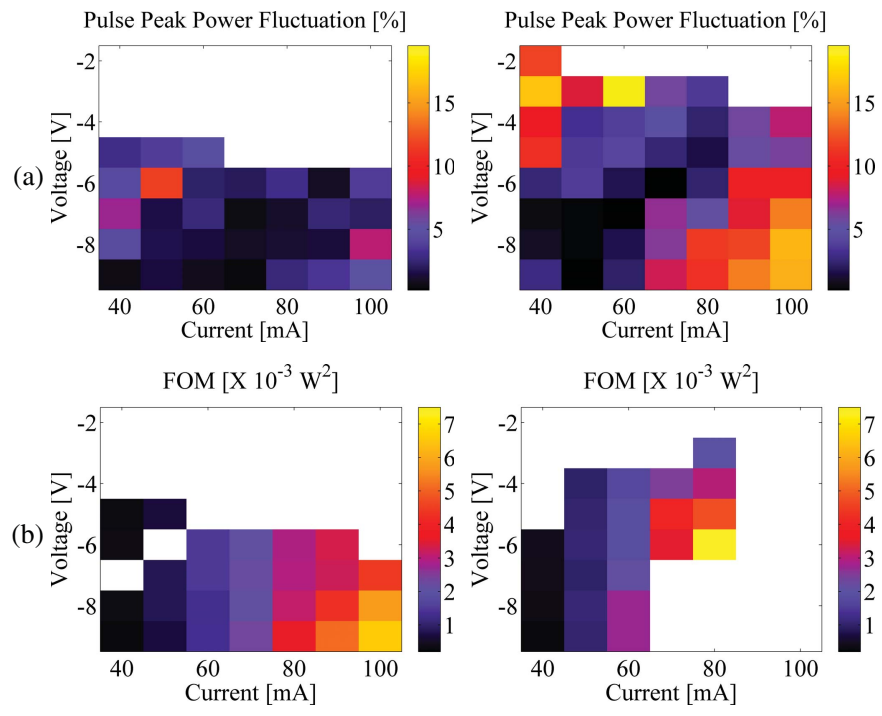

Fig. 5. (a) Maps of the $\%$ pulse peak power fluctuation and (b) FOM maps with peak power fluctuation lower than $5 \%$ for the considered configurations: HR (left) and LR (right).

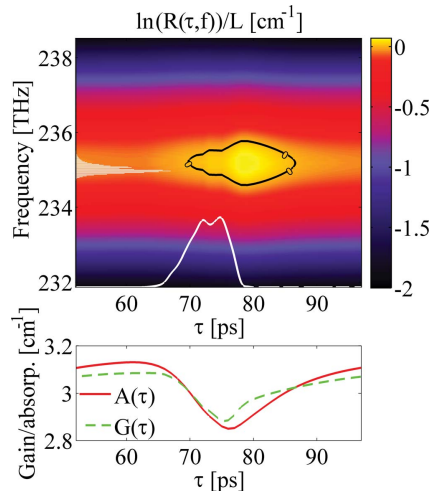

(a)

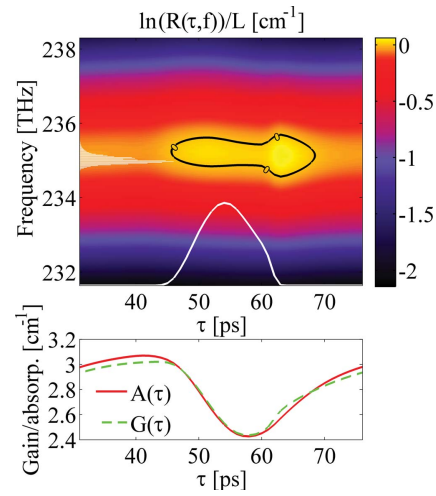

(b)
Fig. 6. Analysis of the ML pulse stability based on the calculation of the round trip gain and loss for the HR design. Map of the normalized round trip gain spectra $\ln (\mathrm{R}(\tau, \mathrm{f})) / \mathrm{L}$ experienced by a ML pulse; the area enclosed by the black line corresponds to the region where the round trip gain $R(\tau, f) \geq 1$. In white, on the left part of the map, the pulse optical spectrum is shown in linear scale and the white line represents the time trace of the pulse power. The lower graph shows the contributions to the total round-trip gain due to amplification in the gain section $\mathrm{G}(\tau)$, and saturable and non-saturable losses in the cavity $\mathrm{A}(\tau)$ evaluated at the peak frequency of the optical spectrum. (a) $I_{g}=40 \mathrm{~mA}$ and $V_{a}=-7 \mathrm{~V}$ (AC width $=8.6 \mathrm{ps}$, FOM $=$ $0.4 \times 10^{-3} \mathrm{~W}^{2}$ ). (b) $I_{g}=80 \mathrm{~mA}$ and $V_{a}=-7 \mathrm{~V}(\mathrm{AC}$ width $=10.7 \mathrm{ps}$, $\mathrm{FOM}=1.5 \times 10^{-3} \mathrm{~W}^{2}$ ).

to the region where the round trip gain $R(\tau, f) \geq 1$ (i.e. $\ln (R(\tau, f)) / \mathrm{L} \geq 0)$.

In addition, the round trip gain evaluated at the peak frequency of the optical spectrum, $f_{\text {peak }}$, has been then decomposed in terms of the overall amplification in the gain section, $\mathrm{G}(\tau)$, and the saturable and non-saturable losses, $\mathrm{A}(\tau)$, experienced by the pulse over a round trip in the cavity, such that $R\left(\tau, f_{\text {peak }}\right)$ can be simply written as $R\left(\tau, f_{\text {peak }}\right)=$ $\exp [\mathrm{G}(\tau) \mathrm{L}-\mathrm{A}(\tau) \mathrm{L}]$. The dynamics of $\mathrm{G}(\tau)$ and $\mathrm{A}(\tau)$ are then shown at the bottom of Fig. 6.

Two ML regimes achieved near the threshold $\left(\mathrm{I}_{g}=40 \mathrm{~mA}\right.$, $\mathrm{V}_{a}=-7 \mathrm{~V}$ with FOM $=0.4 \times 10^{-3} \mathrm{~W}^{2}$, left column of 


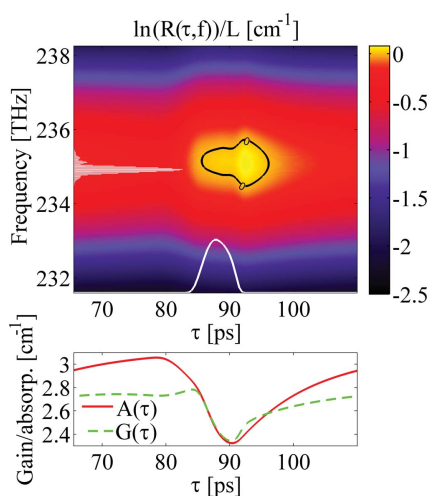

(a)

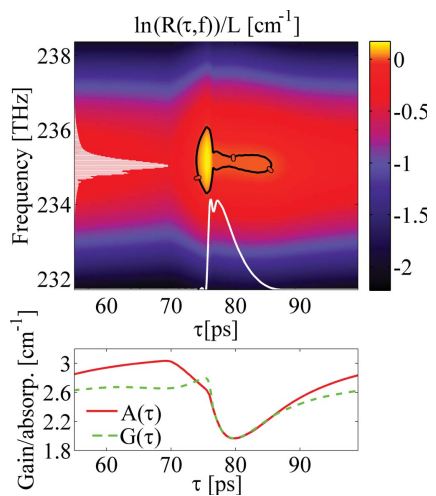

(b)
Fig. 7. As in Fig. 6, analysis of the ML pulse stability for the LR design. (a) $I_{g}=50 \mathrm{~mA}$ and $V_{a}=-6 \mathrm{~V}$ (AC width $=5.1 \mathrm{ps}, \mathrm{FOM}=$

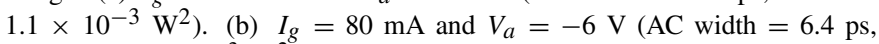
$\left.\mathrm{FOM}=7.3 \times 10^{-3} \mathrm{~W}^{2}\right)$.

Fig. 6) and far above threshold $\left(\mathrm{I}_{g}=80 \mathrm{~mA}, \mathrm{~V}_{a}=-7 \mathrm{~V}\right.$, with $\mathrm{FOM}=1.5 \times 10^{-3} \mathrm{~W}^{2}$ right column of Fig. 6) are considered respectively.

In the ML operation close to threshold, a large region of net gain $R(\tau, f) \geq 1$ extending well beyond the pulse trailing edge is observed. The amplification of the spontaneous emission in this window gives rise to instability which is responsible for the pulse peak power fluctuation (TE instability) shown in Fig. 5 (top) [5]. Moreover, this can also be clarified by the separate contribution of the gain and absorber dynamics on the pulse formation (bottom of Fig. 6). It is evident that $\mathrm{G}(\tau)$ remains higher than $\mathrm{A}(\tau)$ even after the ML pulse, where spontaneous emission noise amplification can occur and an instability is therefore induced at the trailing edge of the pulse. For operation far from threshold (right column of Fig. 6) on the contrary, the net gain window extending beyond the pulse TE is significantly reduced so that no significant instabilities are induced on the ML pulse train.

Regarding the LR geometry, the TE instability is also observed near threshold but for lower reverse voltage where the FOM is poor and comparable to the HR device (Fig. 5). Thus we examine the gain/absorption dynamics for operation at higher current: for $\mathrm{I}_{g}=50 \mathrm{~mA}, \mathrm{~V}_{a}=-6 \mathrm{~V}$ (Fig. 7 left column) and $\mathrm{I}_{g}=80 \mathrm{~mA}, \mathrm{~V}_{a}=-6 \mathrm{~V}$ (Fig. 7, right column). In the first case, the LR device exhibits similar behavior to the HR device at high current $\left(\mathrm{I}_{g}=80 \mathrm{~mA}\right.$, Fig. 6, right column): the ML is stable but with lower FOM $\left(1.1 \times 10^{-3}\right.$ $\mathrm{W}^{2}$ ) than the HR device due to lower average power (operating at lower current). Considering the second case, the LR device exhibits significantly higher FOM than the HR device (7.3 $\times 10^{-3} \mathrm{~W}^{2}$ and $1.5 \times 10^{-3} \mathrm{~W}^{2}$ respectively), however accompanied with a leading edge (LE) instability due to a net gain window at the leading edge of the pulse (Fig. 7, right column). The LE instability is responsible for the increased pulse peak power fluctuation in this regime (Fig. 5, right column). Although under the specific conditions the operation is stable, with further increase of $\mathrm{I}_{g}$ the ML exhibits large fluctuations ( $>10 \%$ peak power) and tends to fall in an unstable regime.
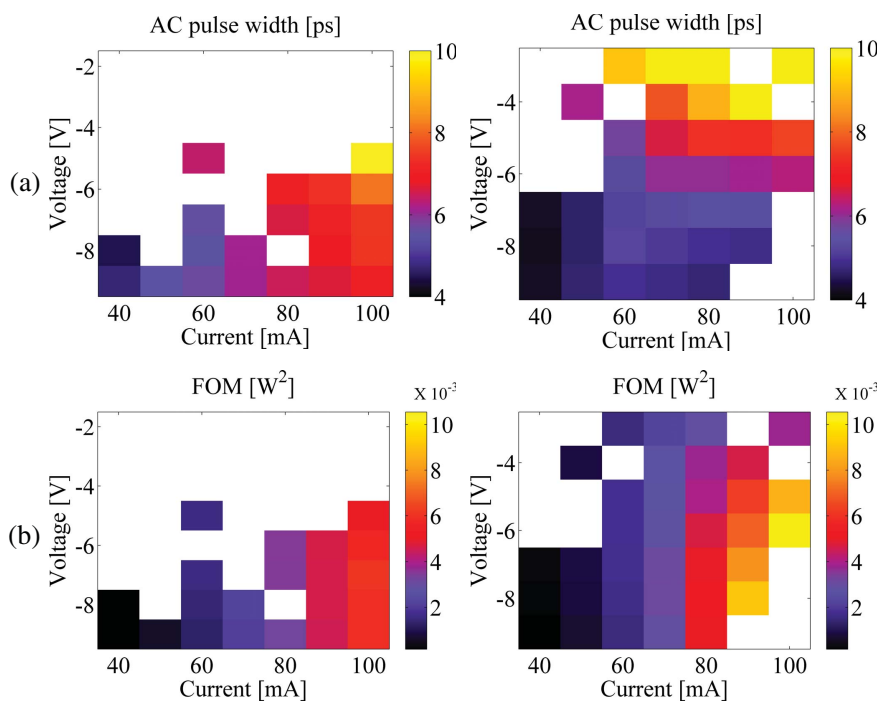

Fig. 8. (a) Maps of pulse AC width and (b) FOM for absorber length $L_{a}=0.35 \mathrm{~mm}$ and peak power fluctuation lower than 5\%: HR (left) and LR (right).

To conclude the results regarding the comparison of the HR and LR devices, the LR device shows a larger FOM in all cases, but the largest values are obtained where the ML is highly unstable (high voltage and high current). At low currents the LR device favors significantly shorter pulses, but with lower average power, thus the FOM remains comparable to the HR case. At high voltage and current where the FOM is much larger for the LR device, the LE instability in the ML regime is the actual limit for the maximum achievable FOM. The LE instability in the HR coated SA configuration, seems instead to start at higher current above threshold.

We must point out that simulation results described above differ significantly from the operating regimes reported in [4] for QW ML lasers where the pulse shortening was accompanied by an improved ML stability over a wide range of gain currents in the LR geometry with respect to the HR geometry. We think that this discrepancy is strictly related to the peculiar differences between the non-linear gain and absorption dynamics governing the ML regimes in QW and QD ML lasers respectively (large differences in gain and absorption saturation energies and recovery times are expected).

Furthermore, in the next section we show that the performances of the LR geometry with respect to the HR one are very sensitive to the actual cavity design and in particular to the absorber to gain section length ratio.

\section{Optimization of the LR Configuration}

From the findings of the previous paragraph, it is obvious that the advantage of higher FOM of the LR design is downgraded by unstable operating regimes and large amplitude noise. Thus optimization of the design is required in order to fully exploit the advantages of the LR case. The solution may rely on a structure with different saturation characteristics. As shown in [9], the effect of the leading edge instability which appears at high current can be minimized if the length of the absorber is increased. In order to confirm the validity of this 


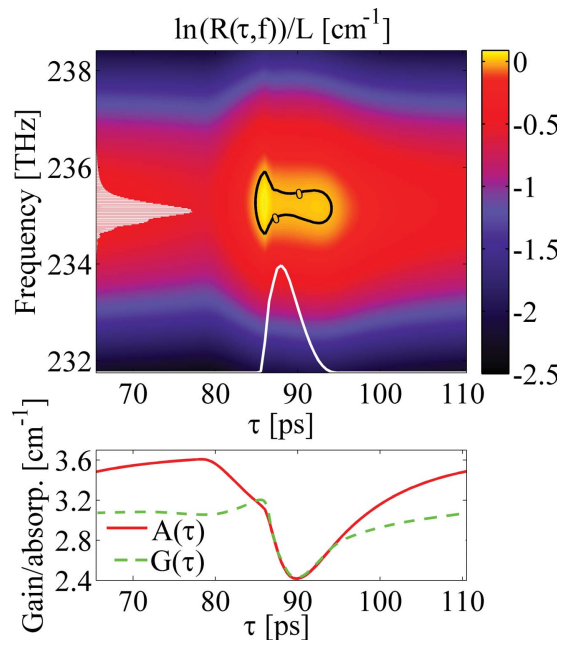

Fig. 9. As in Fig. 5, analysis of the ML pulse stability for the LR design in the case of a $0.35 \mathrm{~mm}$ long absorber. $I_{g}=80 \mathrm{~mA}$ and $\mathrm{Va}=-7 \mathrm{~V}$ $\left(\mathrm{AC}\right.$ width $\left.=5.5 \mathrm{ps}, \mathrm{FOM}=5 \times 10^{-3} \mathrm{~W}^{2}\right)$.

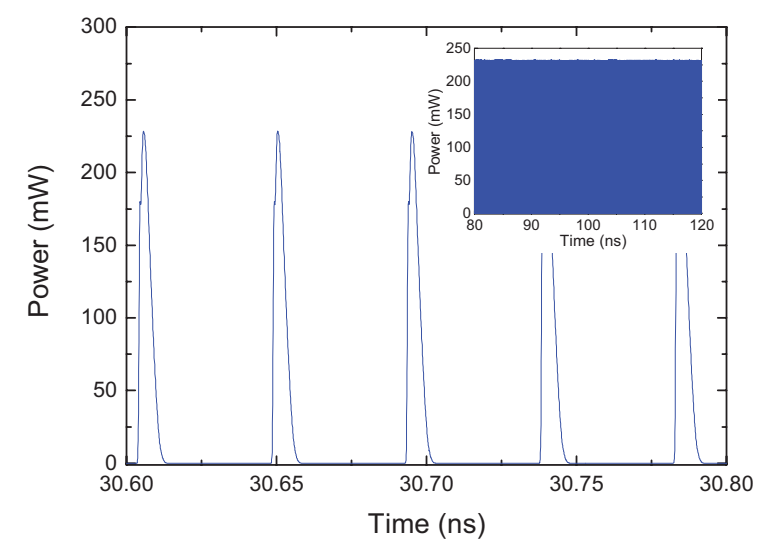

Fig. 10. Output pulse train for the LR device with absorber length $L_{a}=0.35 \mathrm{~mm}$ for $I_{g}=80 \mathrm{~mA}$ and $V_{a}=-7 \mathrm{~V}$.

claim for the case we study, we mapped the operation of both the HR and LR devices with a longer absorber $(0.35 \mathrm{~mm})$ while keeping the same length of the cavity $(2 \mathrm{~mm})$.

Fig. 8 shows a map of the FOM only for the conditions where the peak power fluctuation is below $5 \%$. It is evident that the LR geometry now favors higher FOM in the majority of stable ML operation, and especially at high currents. This is because the pulse leading edge instability (LE) which is detrimental for the LR geometry with the short absorber $(0.3 \mathrm{~mm}$, Fig. 5) occurs at higher current values for the device with the long absorber $(0.35 \mathrm{~mm})$. This effect is highlighted in Fig. 9 which illustrates the stability maps and the gain/absorption dynamics, for $\mathrm{I}_{g}=80 \mathrm{~mA}, \mathrm{~V}_{a}=-7 \mathrm{~V}$ resulting in a AC width of $5.5 \mathrm{ps}$ and FOM of $5 \times 10^{-3} \mathrm{~W}^{2}$. A pulse train in two different scales is shown in Fig. 10 for the same conditions. Further optimization of the LR design with increasing even more the length of the absorber may be detrimental for the ML operation, since trailing edge (TE) instability appears near the ML threshold regime [9]. In addition, the high-FOM advantage could vanish since a device with a longer absorber exhibits more losses.
Apart from the optimization based on the saturable absorber length, devices with different growth conditions have been simulated, assuming different number of QD layers and different QD surface density. The simulations for devices using all the parameters of Table I and assuming 10 QD layers and $0.3 \mathrm{~mm}$ long absorber showed similar results with the ones reported in paragraphs A and B: there is no significant performance improvement in terms of noise and stability using the LR device compared to the AR device. On the other hand, simulations assuming devices with 5 QD layers, $0.35 \mathrm{~mm}$ long absorber and an increased QD density of $\mathrm{N}_{d}=4 \times 10^{14} \mathrm{~m}^{-2}$ exhibit for the LR device a wider range of stable ML operation and higher FOM, compared to the HR device; however, both devices under these parameters give a reduced range of stable ML operation due to high amplitude noise; An exhaustive comparison of ML performance between the LR and HR configurations for the entire set of the structural parameters cannot be performed in the context of the present work. However, based on the available results we can estimate that the LR configuration leads to an improvement in the device performance under certain conditions: long enough absorber, moderate value of QD density and small number of QD layers.

\section{CONCLUSION}

The properties of an alternative design for two-section mode-locked quantum dot lasers have been analyzed theoretically. We compare the operation of devices with the absorber section located near the low-reflection facet with the conventional devices with the absorber near the highreflection facet. The analysis is carried out by means of a bidirectional FDTW model which properly accounts for the quantum dot material system and the colliding effects in the absorber section. We show that the devices based on the alternative design prevent the formation of the colliding effects and generate shorter ML pulses with higher peak power than the devices based on the conventional design which exploits the self-colliding effects. Although the maximum achieved peak-with-average power product is limited by increased noise and unstable regimes related to instabilities, with optimization of the design, stable and low noise ML operation with superior performance can be achieved.

\section{REFERENCES}

[1] M. G. Thompson, A. R. Rae, M. Xia, R. V. Penty, and I. H White, "InGaAs quantum-dot mode-locked laser diodes," IEEE J. Sel. Topics Quantum Electron., vol. 15, no. 3, pp. 661-672, May-Jun. 2009.

[2] E. U. Rafailov, M. A. Cataluna, and W. Sibbett, "Mode-locked quantumdot lasers," Nature Photon., vol. 1, pp. 395-401, Nov. 2007.

[3] E. A. Avrutin, J. H. Marsh, and E. L. Portnoi, "Monolithic and multigigahertz mode-locked semiconductor lasers: Constructions, experiments, models and applications," IEE Proc.-Optoelectron., vol. 147, no. 4, pp. 251-278, Aug. 2000.

[4] J. Javaloyes and S. Balle, "Anticolliding design for monolithic passively mode-locked semiconductor lasers," Opt. Lett., vol. 36, no. 22, pp. 44074409, 2011.

[5] M. Rossetti, P. Bardella, and I. Montrosset, "Time-domain travellingwave model for quantum dot passively mode-locked lasers," IEEE J. Quantum Electron., vol. 47, no. 2, pp. 139-150, Feb. 2011. 
[6] A. Markus, M. Rossetti, V. Calligari, D. Chek-Al-Kar, J. X. Chen, A. Fiore, and R. Scollo, "Two-state switching and dynamics in quantum dot two-section lasers," J. Appl. Phys., vol. 100, no. 11, pp. 113104-1113104-5, Dec. 2006.

[7] M. Rossetti, P. Bardella, and I. Montrosset, "Modeling passive modelocking in quantum dot lasers: A comparison between a finite-difference traveling-wave model and a delayed differential equation approach," IEEE J. Quantum Electron., vol. 47, no. 5, pp. 569-576, May 2011.

[8] M. Radziunas, A. G. Vladimirov, E. A. Viktorov, G. Fiol, H. Schmeckebier, and D. Bimberg, "Pulse broadening in quantum-dot mode-locked semiconductor lasers: Simulation, analysis, and experiments," IEEE J. Quantum Electron., vol. 47, no. 7, pp. 935-943, Jul. 2011.

[9] M. Rossetti, T. Xu, P. Bardella, and I. Montrosset, "Impact of gain saturation on passive mode locking regimes in quantum dot lasers with straight and tapered waveguides," IEEE J. Quantum Electron., vol. 47, no. 11, pp. 1404-1413, Nov. 2011.

Hercules Simos received the B.Sc. degree in informatics, the M.Sc. degree in telecommunications engineering, and the Ph.D. degree with research on photonic devices for all-optical wavelength conversion and regeneration subsystems for WDM telecommunication networks from the University of Athens, Athens, Greece, in 1998, 2000, and 2007, respectively.

$\mathrm{He}$ was with the Optical Communications Laboratory, University of Athens, from 1999 to 2012, as a Research Associate of national and European projects on optical communications. Since 2012, he has been a Lecturer with the Technical Educational Institute of Piraeus, Piraeus, Greece. He has experience in the modeling and experimental investigation of semiconductor lasers, amplifiers, and related subsystems. His current research interests include semiconductor laser dynamics, and photonic devices for optical networks, optical signal processing, and sensing.

Mattia Rossetti received the Laurea Magistrale degree in physical engineering and the $\mathrm{Ph}$. D. degree in electronic and communication engineering from the Politecnico di Torino, Turin, Italy, in 2007 and 2011, respectively.

He is currently a Post-Doctoral Fellow with the Politecnico di Torino. His current research interests include modeling and simulation of quantum-dotbased optoelectronic devices.

Christos Simos received the B.Sc. degree in physics from the University of Athens, Athens, Greece, the DEA degree in high-frequency electronics and optoelectronics, and the Ph.D. degree in photonics from the University of Limoges, Limoges, France, in 1995, 1996, and 2002 respectively.

He was a Post-Doctoral Research Fellow with the Laboratoire des Propriétés Optiques de Matériaux \& Applications, Angers, France, from 2002 to 2003. From 2003 to 2006, he was a Senior Scientist with Forth Photonics S.A., Athens, Greece. He is currently a Lecturer with the Department of Electronics, Technological Educational Institution of Lamia, Lamia, Greece, and a Research Collaborator with the Photonics Technology Laboratory, National and Kapodistrian University of Athens, Athens. He has co-authored more than 50 papers in journals and international conferences. His current research interests include quantum-dot lasers, nonlinear optical properties of novel materials, laser systems and applications, and optoelectronic systems for metrology and medical diagnostics.
Charis Mesaritakis received the B.S. degree in informatics, the M.S. degree in microelectronics, and the Ph.D. degree in quantum-dot mode-locked lasers for telecommunication and biomedical applications from the National and Kapodistrian University of Athens, Athens, Greece, in 2004 and 2011, respectively.

$\mathrm{He}$ is currently a Post-Doctoral Researcher with the Optical Communications Laboratory, Department of Informatics and Telecommunications, National and Kapodistrian University of Athens. His current research interests include quantum-dot semiconductor laser dynamics, single-photon emitters, optical sensors, and microring resonators.

Tianhong Xu received the B.Sc. degree in electronic science and technology from the Huazhong University of Science and Technology, Wuhan, China, in 2007, and the Laurea Magistrale degree in electronic and communication engineering from the Politecnico di Torino, Turin, Italy in 2009, where she is currently pursuing the Ph.D. degree.

Her current research interests include modeling and simulation of quantumdot-based optoelectronic devices.

Paolo Bardella received the Laurea degree in electronic engineering and the Ph.D. degree in electronic and communication engineering from the Politecnico di Torino, Turin, Italy, in 2001 and 2006, respectively.

$\mathrm{He}$ is currently a Post-Doctoral Fellow with the Dipartimento di Elettronica e Telecomunicazioni, Politecnico di Torino, where he is involved in research on the simulation of multisection semiconductor lasers in mode-locking and self-pulsating regime. His current research interests include modeling and design of multisection quantum-dot semiconductor lasers, and superluminescent diodes.

Ivo Montrosset (M'92) has been a Professor of optoelectronics with the Politecnico di Torino, Turin, Italy, since 1990. His current research interests include guided wave-optics, solid-state and semiconductor lasers, and other related topics.

$\mathrm{He}$ is a member of the IEEE Photonics Society.

Dimitris Syvridis received the B.Sc. degree in physics, the M.Sc. degree in telecommunications, and the Ph.D. degree in physics in 1982, 1984, and 1988, respectively.

He was a Researcher with the Electronics Laboratory, National and Kapodistrian University of Athens, Athens, Greece, from 1988 to 1990. From 1990 to 1993, he was a Researcher with the Institute of Quantum Electronics, Swiss Federal Institute of Technology (ETH Zurich), Zurich, Switzerland. From 1993 to 1997, he was a Senior Researcher with the Department of Informatics, National and Kapodistrian University of Athens, where he is currently a Full Professor. He has participated in many European research projects on optical communications. He has authored or co-authored more than 130 papers in scientific journals and conference proceedings. His current research interests include optical communications and networks, photonic devices, and related subsystems, as well as photonic integration.

Dr. Syvridis is a reviewer for journals of the IEEE. 